\title{
EXPERIMENTAL ASBESTOSIS IN RATS THE EFFECT OF PARTICLE SIZE AND OF ADDED ALUMINA BY
}

\author{
J. MACLEAN SMITH, I. D. P. WOOTTON, AND E. J. KING \\ From the Postgraduate Medical School of London
}

(RECEIVED FOR PUBLICATION FEBRUARY 28, 1951)

An antidotal effect on silicosis was described by Denny, Robson, and Irwin (1937), when rabbits exposed to silica dust were treated with aluminium inhalations. It seemed important, therefore, to determine whether there was any such beneficial effect from alumina in asbestosis. Experiments have been designed to test this in the rat by the intratracheal injection of the dust with and without alumina. Experiments with rabbits have already been published (King, Clegg, and Rae, 1946).

\section{Methods}

Rhodesian asbestos dust was prepared by Messrs. Turner Brothers to yield an average fibre length of $2.5 \mu$ (referred to as "short fibre") in one sample, and $15 \mu$ (referred to as "long fibre") in another. Alumina was prepared according to the directions of Gardner, Dworski, and Delahant (1944). The depression of quartz solubility studied immediately before use showed that $1 \%$ of alumina reduced the silica solubility of asbestos from 5.15 mg. to $0.285 \mathrm{mg}$. $\mathrm{SiO}_{2}$ per $100 \mathrm{ml}$.

The results of previous experiments involving the administration of asbestos and alumina suggested that a useful index of pathogenicity could be found by considering the survival times of experimental animals. In these former experiments all rats receiving one particular type of dust were confined together in one cage. It was evident that factors affecting single cages, for example epidemic infections, were obscuring differences in survival times produced by the different injections. For this reason the present experiment was arranged so that these extraneous causes of death would affect all the groups equally.

A homogeneous group of 150 young male rats of the Medical Research Council hooded black and white strain was taken. They were divided at random into five subgroups of 30. Each subgroup then received intratracheal injections of one of the following: long fibre asbestos, short fibre asbestos, long fibre asbestos and alumina, short fibre asbestos and alumina, alumina alone. All the work of injecting was completed in 48 hours.

Sterilized suspensions of $10 \mathrm{mg}$. of asbestos dust in $1 \mathrm{ml}$. of normal saline were injected intratracheally in anaesthetized animals (ether), or this was given with $1 \mathrm{mg}$. of alumina added, and the final series of rats was treated with $10 \mathrm{mg}$. alumina alone.

After injection each rat was marked by ear-clipping, and any animals dying postoperatively were replaced. The rats were then distributed into 15 cages, so that each cage contained two members of each subgroup; the cages were housed in a single animal room. The survival time of each rat was then noted in days from the time of injection.

Survival Results

The survival times of the various subgroups are plotted in Fig. 1. At the end of the experiment a few remaining survivors were killed. 


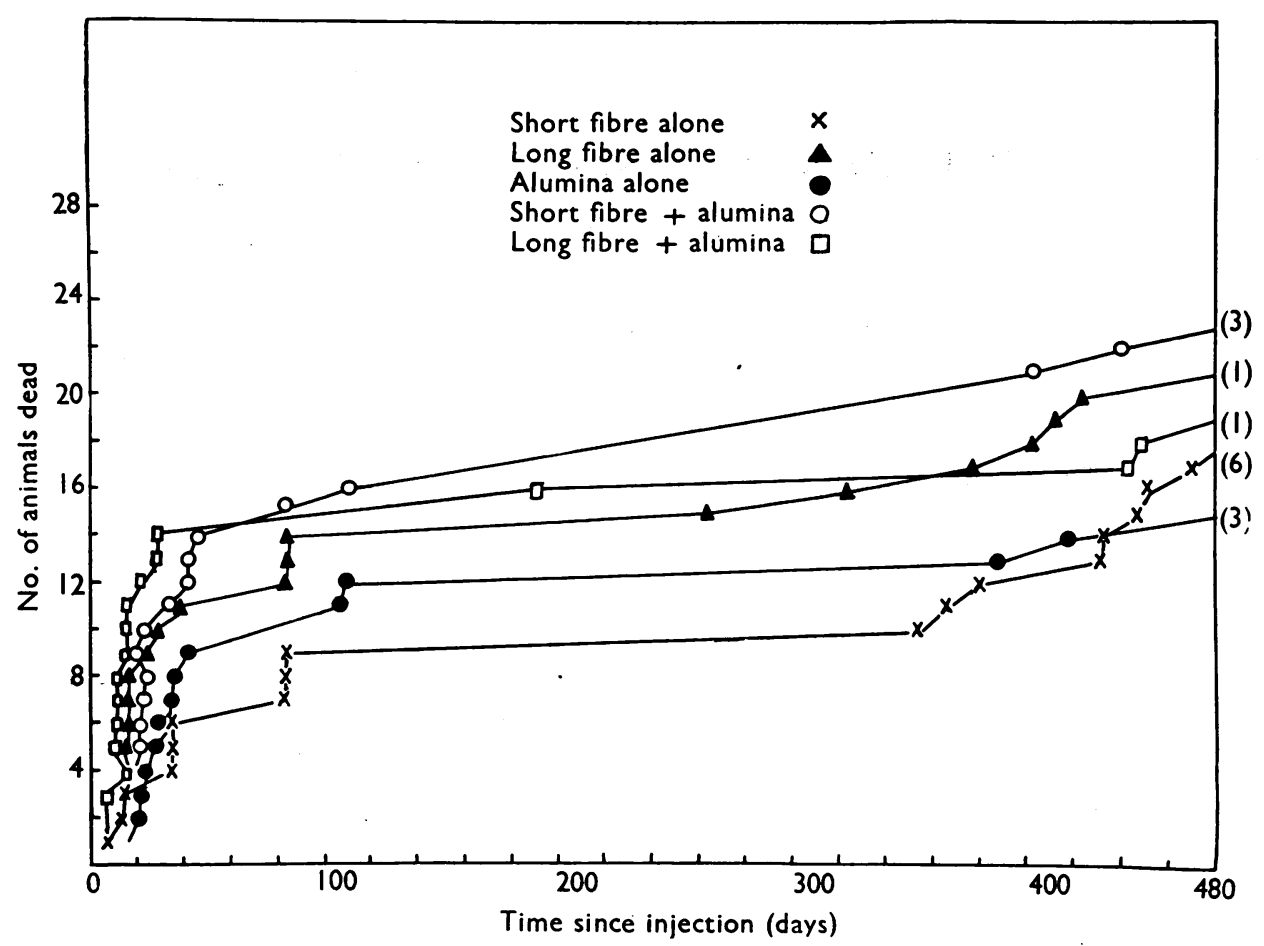

FIG. 1.-Survival of rats with experimental asbestosis. The number in parentheses at the end of each curve represents the number of survivors in each group.

An inspection of the broad trends will show that convincing differences between the different subgroups are not shown. Two periods of frequent deaths were separated by an interval when relatively few deaths occurred. The same general shape is shown by all the subgroups, and it is apparent that the same factors were affecting all the cages in the experiment. It seems, then, that under the living conditions of this experiment the supposedly small differences between subgroups are lost in relatively large irrelevant causes of death. The nature of these is presumably infective.

\section{The Histology of the Lungs}

The lungs of all animals were fixed in $10 \%$ neutralized formol saline and were stained by haematoxylin and eosin and by a silver impregnation method for reticulin. Representative slides from each group were also stained by Van Gieson's method for fibrous tissue.

The slides were examined without knowledge of the group from which they came, and the histological assessment was recorded before the groups were reassembled. Some animals were lost by cannibalism; others died and became decomposed during week-end periods and were not suitable for histological examination.

Short Fibre Asbestos.-The presence of short fibre asbestos in the lungs for periods up to 483 days in no case produced more than moderate peribronchial fibrosis; this was seen in all 20 animals examined. Slight interstitial fibrosis was present in only three animals. Two animals, dying within a few days of injection, showed 
intra-alveolar inflammatory exudate ; one animal died of bronchopneumonia on the 17 th day and one other, which died on the 18th day, showed purulent bronchitis. Only one animal developed pleurisy. Fourteen rats showed a moderate degree of emphysema. The lungs were not congested. Many of the animals developed hyperplasia of the peribronchial lymph nodes, and all those surviving more than a fortnight showed yellow pigmented phagocytes in varying numbers. Giant cells were rarely seen and true asbestos bodies were not found. In four cases "sausage bodies," similar to those described by Gloyne as precursors of the asbestos body, were present in the alveolar phagocytes of the animals which were examined 434 days after injection. These were not fully developed asbestos bodies, as they did not show segmentation, crenation, or bulbous ends (Gloyne, 1938). Intracellular and extracellular black and brown pigment was common. Infarcts were present in the lungs of four animals which died on the second, 17th, 21 st, and 483rd days, but there was nothing to suggest the cause of these lesions. These histological appearances are shown in Figs. 2 and 3. One animal (killed on the 483rd day) had a small $(2 \mathrm{~mm}$.) non-malignant adenoma. As there were no other adenomata in other animals exposed for the same period, this was considered to be an intercurrent disease. In all, 20 animals in this group were examined.

Long Fibre Asbestos.--In most of the 14 animals examined (except those dying shortly after injection), which had received long fibre asbestos, moderate interstitial fibrosis was present (Figs. 4 and 5). This was of a scattered distribution, often starshaped, and of grade 1 severity (Belt and King, 1945). The basis of this fibrosis, as has been pointed out by previous workers, appeared to be a patchy alveolar collapse, which was best seen in the haematoxylin and eosin preparations of those dying in the first three weeks. One animal died of bronchopneumonia seven days after injection. Three of the rats surviving over 350 days showed no fibrosis; two of these animals had lung infarcts and one had purulent bronchitis. Pigmented phagocytes were common in the older animals. Including the two already mentioned, infarcts were present in four of the animals and another showed an area of collapse. Dust cells were common, but giant cells were not seen. Emphysema of moderate degree was common.

Alumina.-No fibrosis was present after the injection of alumina except in one case. This showed mild intersititial fibrosis in one lung only (confirmed in the Van Gieson preparation). Animals dying within three weeks of injection showed inflammatory alveolar exudate: all the lungs showed noticeable vascularity. Emphysema was not found, and the only infectious complication was purulent bronchitis in one rat which died nine days after injection. Intra-alveolar collections of dust cells and also interstitial mononuclear cells were prominent in many of the animals. In general, the alumina caused increased vascularity without significant scarring in the 14 rats examined (Figs. 6 and 7).

Short Fibre Asbestos Plus Alumina.-The addition of alumina to the short fibre asbestos produced an interesting change (Figs. 8 and 9). In six of the 11 animals patchy atelectasis of the alveoli occurred and was associated with scattered interstitial fibrosis, similar to that seen in the animals receiving long fibre. A slight degree of emphysema was found in a few animals, and one died of bronchopneumonia. Again, there were a few cases of peribronchial lymphatic hyperplasia. 


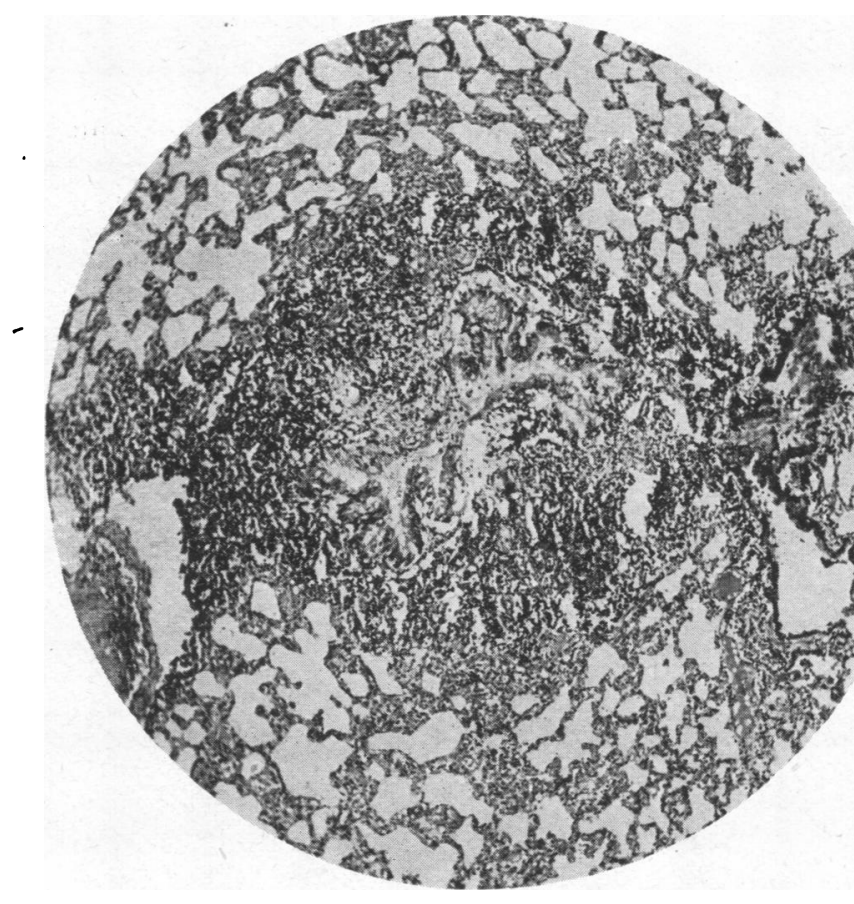

Fig. 2

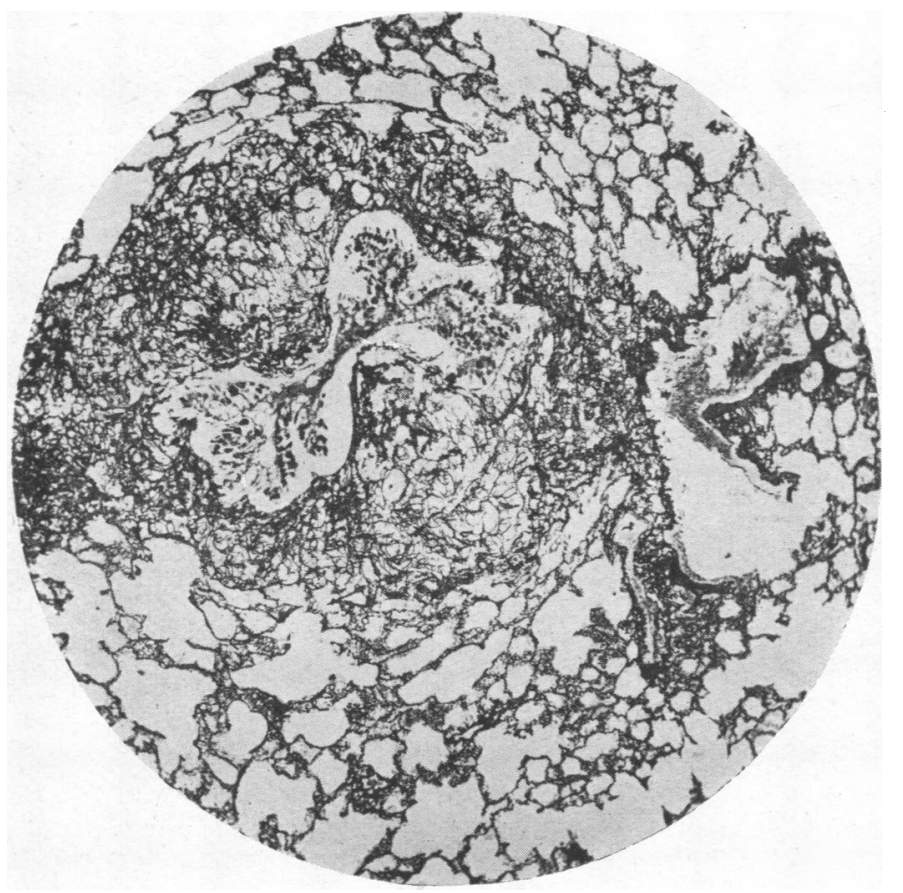

FIG. 2.-Short fibre asbestos: 483 days' survival. There is a little increase in the supporting connective tissue around a bronchus. Haematoxylin and eosin stain $(X$ 50).

FIG. 3.-Same case as Fig. 2, but impregnated with silver to show the fibrous nature of tissue around the bronchus $(\times 50)$.

FIG. 3 


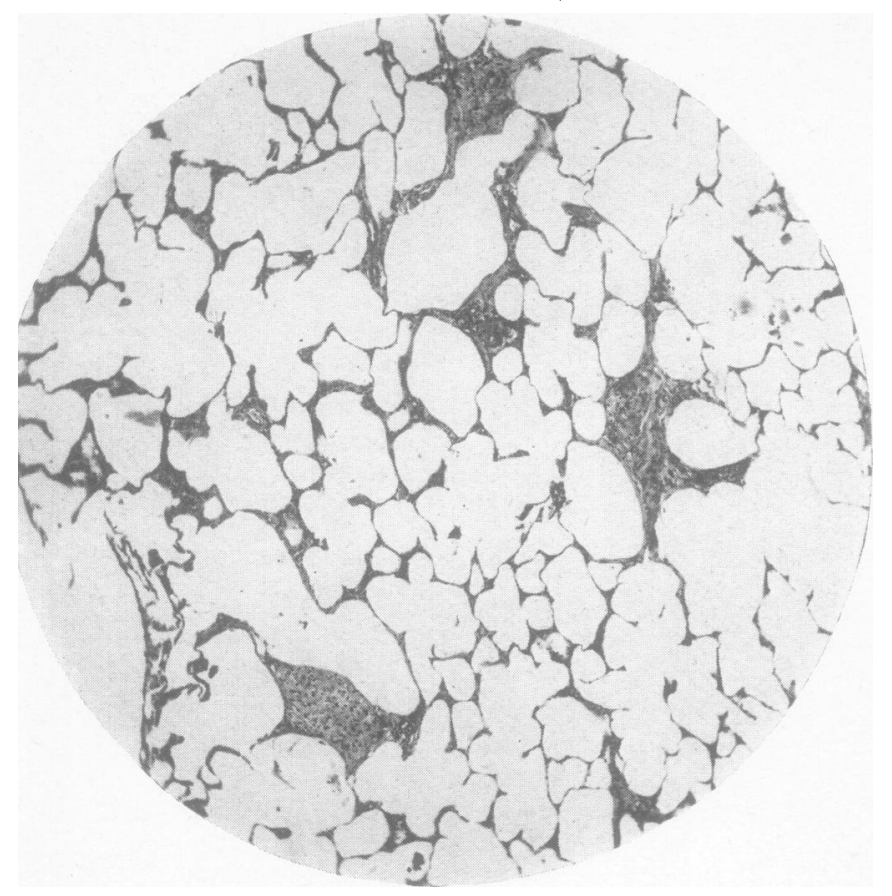

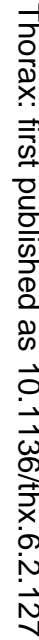

FIG. 4

Fig. 4.-Long fibre asbestos: 483 days' survival. Section of lung showing small starshaped foci of consolidation. Haematoxylin and eosin stain $(\times 45)$.

FIG. 5.-Same as Fig. 4, but impregnated with silver to show that the solid foci are fibrous $(\times 45)$.

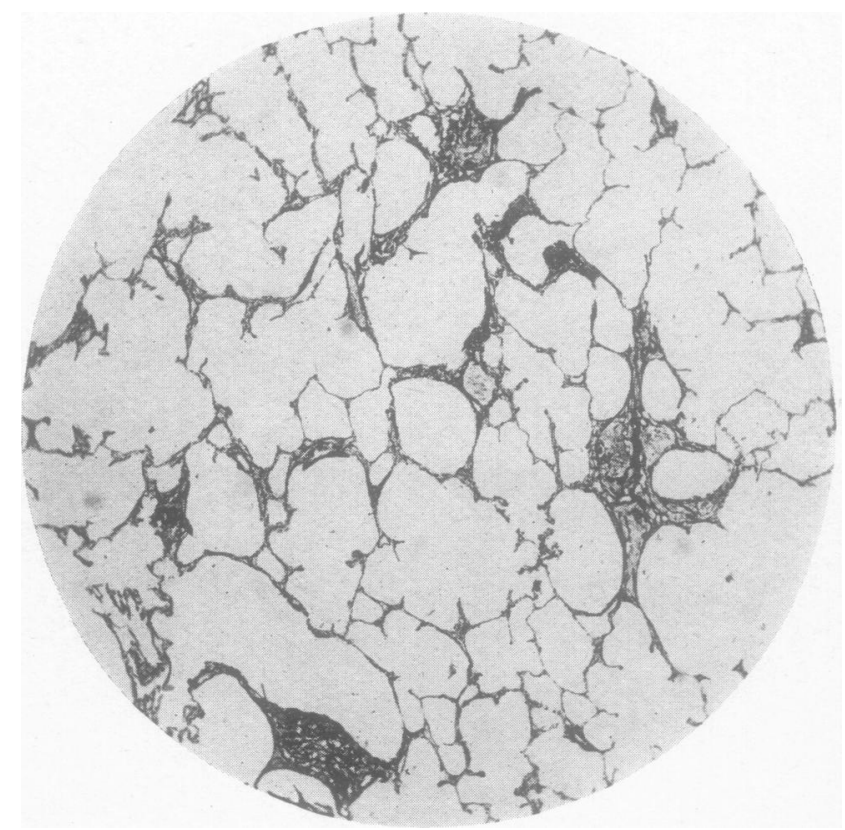

Fig. 5 


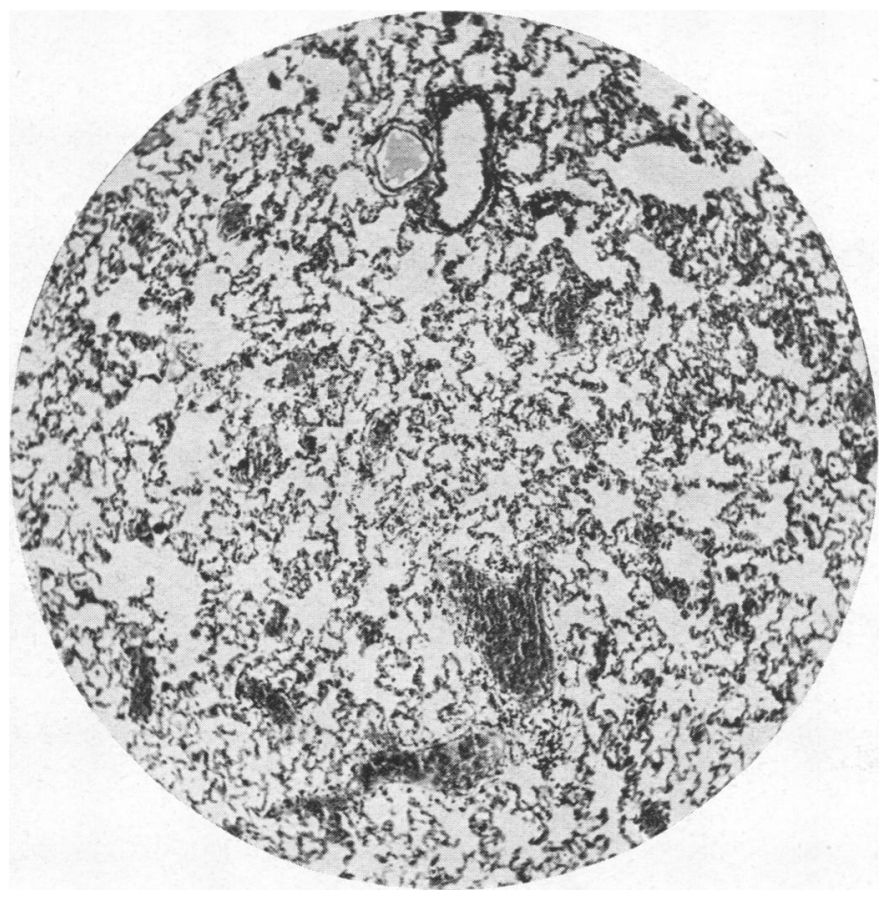

FIG. 6.-Alumina alone: 483 days' survival. The walls of the air sacs are rather thick and engorged with blood, but there is no significant abnormality. Haematoxylin and eosin stain $(\times 50)$.

Fic. 7.- Same as Fig. 6, but impregnated with silver, and shows the normal fibrous framework of the rat's lung $(\times 50)$.

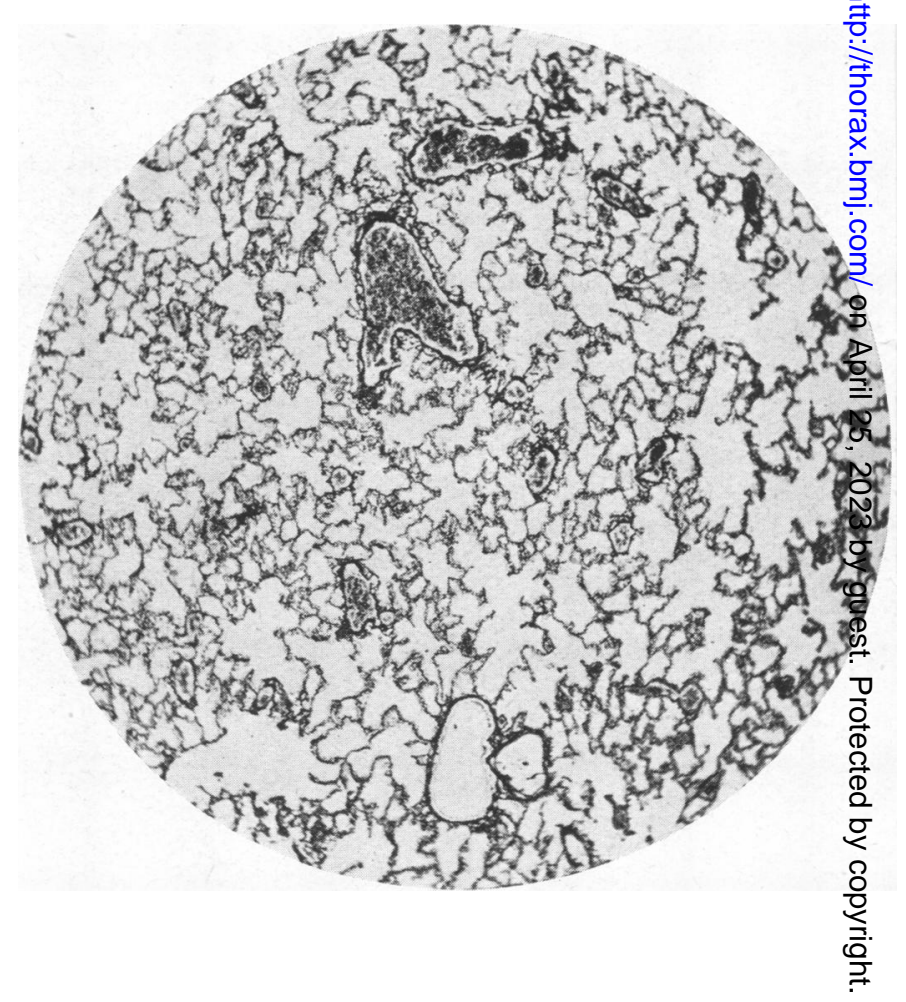


FIG. 8.-Short fibre asbestos and alumina: 483 days' survival. Scattered foci of consolidation are seen both around the bronchi (upper left) and in the lung tissue. Haematoxylin and eosin stain ( $X$ 55).

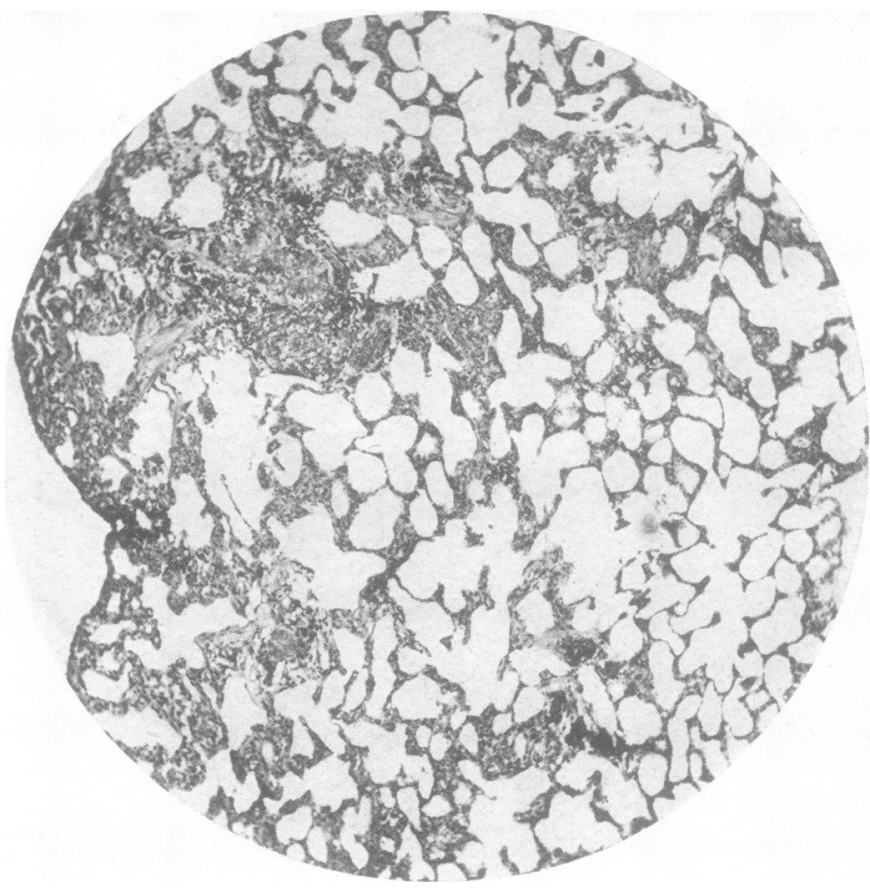

FIG. 9.-Same as Fig. 8, but impregnated with silver to show that the consolidation is largely fibrous ( $\times 55)$. 


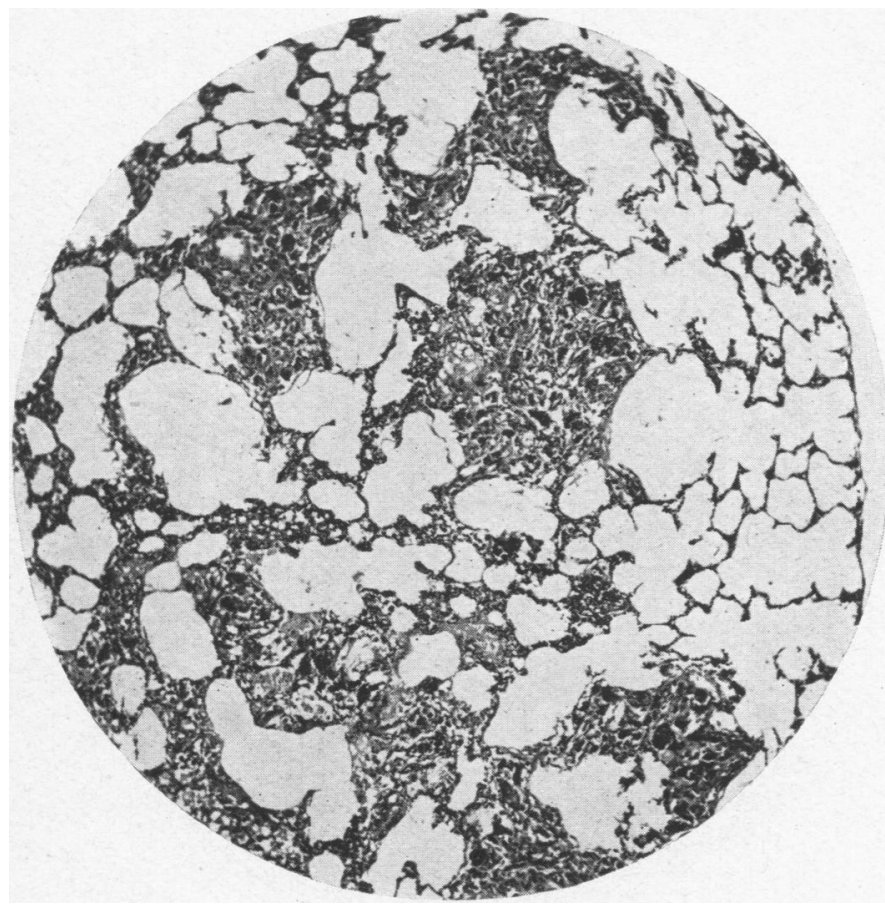

Fig. 10.-Long fibre asbestos plus alumina: 483 days' survival. Fairly numerous foci of consolidation are seen in the lung substance. This case happens to be more severe than that shown in Figs. 4 and 5. Haematoxylin and eosin stain $(X$ 50).

FIG. 11.-Same case as Fig. 10, but impregnated to show that the consolidation is ftbrous $(\times 50)$.

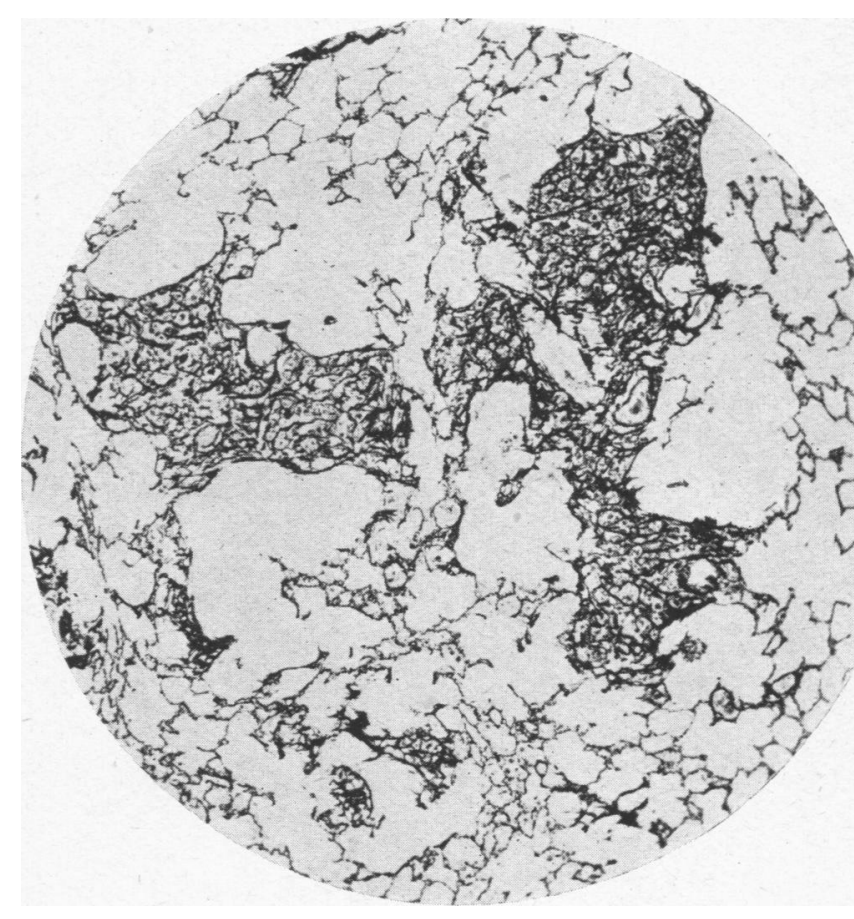


Pigmented phagocytes occurred as before, but nothing resembling asbestos bodies was seen. Dust cells were common, and in one case were found in the tracheobronchial lymph nodes. Two of the lungs showed apical collapse, and one a large infarct.

Long Fibre Asbestos plus Alumina.-The addition of alumina did not affect the type of fibrosis markedly. If anything, it slightly increased the amount. Some of the animals showed a slight venous congestion. Four of the lungs had areas of collapse, but there were no infarcts. Dust cells were common, but asbestos bodies and giant cells were not seen (Figs. 10 and 11).

In many of the animals of all the above groups it was difficult to recognize the asbestos. In no case was there evidence of silicosis, of squamous metaplasia of the bronchial mucosa, or of tuberculosis. Some of the arterioles showed periarteritis and medial hyperplasia. The results are summarized in Table I.

TABLE I

Summary of Histological Findings

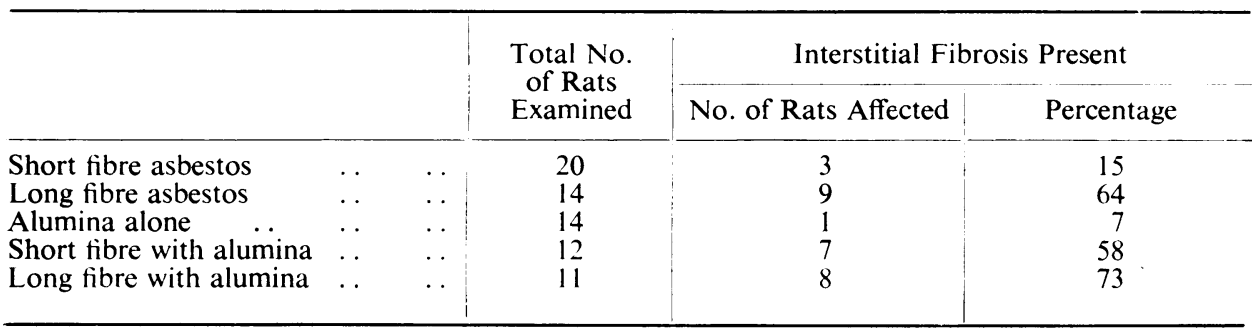

\section{DiscusSION}

Asbestosis in man produces a well-marked, diffuse interstitial fibrosis associated with some chronic bronchitis and emphysema. Dense fibrous pleurisy is common. Golden-yellow foreign bodies named asbestos bodies are found in the alveoli, free or in clumps, in phagocytes or in giant cells. These changes have been described by Lanza (1938). McDonald (1927) described the fibrosis as being fine, diffuse, and different from the whorl-like lesions of silicosis. He noted endarteritis of the small pulmonary arteries, some of which were thrombosed, and also numerous foci of lymphocytes amongst the fibrous tissue. Various authors have remarked that the fibrosis appears in the peribronchial regions and leads to segmental collapse of the lung, which becomes organized and results in a stellate area of fibrous tissue.

The preceding description of the lesions in rats which have been experimentally exposed to asbestos dust shows that, while peribronchial fibrosis is produced by small fibre particles, the stellate areas of collapse and subsequent fibrosis do not appear until large fibre asbestos is used. This last-mentioned lesion is, however, produced when alumina is added to the short fibre inoculum, and it seems reasonable to conclude that an aggregation of materials occurs resulting in an increased particle size which is then able to cause alveolar blockage with stellate collapse and fibrosis. This confirms the report by Gardner (in Lanza, 1938) that lung damage occurs in asbestosis only when there are large particles. The experimental disease differs in that true asbestosis bodies are not formed, and pleurisy only rarely shows. 
Asbestosis bodies have been reported in guinea-pigs and wild rats (Mavrogordato, cited by Simson, 1929 ; Gardner and Cummings, 1931 ; Giroux, 1947), but they were very scanty, and were atypical. They were not found in dogs (Schuster, 1931), or in the tissue culture experiments of Belt, Friedmann, and King (1947). The experimental disease does, however, reproduce the scattered interstitial pulmonary fibrosis, chronic bronchitis, emphysema, scattered foci of lymphocytes, and arteritis with infarcts.

Alumina, when administered to rats by intratracheal injection, produces vascular engorgement and numerous phagocytes, but in the present experiment fibrotic change was caused in only one rat. When added to asbestos it leads to fibrotic changes in some rats receiving small fibre asbestos, which alone is not fibrogenic, and it shows a slight tendency to increase the lesions normally produced by long fibre asbestos. It does not in any way produce an amelioration of the disease as has been described in silicosis.

These results confirm the findings of King, Clegg, and Rae (1946) that the addition of metallic aluminium to the dust appears to be entirely without benefit in asbestosis, and, in fact, increases the reaction. Our results differ in that short fibre asbestos produced practically no reaction, except locally around the bronchi, whereas King, Clegg, and Rae (1946) found that in rabbits a diffuse interstitial fibrosis was produced. This may be a species difference.

\section{SUMmaRY}

A study has been made of the effect of particle size on the production of lung fibrosis in rats by asbestos. Short fibres asbestos $(2.5 \mu)$ caused only a moderate peribronchial fibrosis, whereas long fibre asbestos $(15 \mu)$ caused well-marked interstitial fibrosis resembling that seen in the human disease.

Alumina injected together with asbestos of different particle size increased the fibrosis produced.

The expenses of this investigation were defrayed from a grant received from Messrs. Turner Brothers Asbestos Company, to whom we should like to express our sincere gratitude. For much helpful consultation and advice we are indebted to Dr. C. V. Harrison, Dr. E. R. A. Merewether, Mr. R. G. Soothill, and Mr. E. L. Dawson. We are grateful to Mr. W. Weedon for technical assistance, and to Mr. C. G. Johnston for cutting and staining the sections. The photomicrographs were made by Mr. E. V. Wilmott.

\section{REFERENCES}

Belt, T. H., Friedmann, I., and King, E. J. (1947). J. Path. Bact., 59, 159.

- and King, E. J. (1945). Spec. Rep. Ser. med. Res. Coun., Lond., No. 250, p. 29.

Denny, J. J., Robson, W. D., and Irwin, D. A. (1937). Canad. med. Ass. J., 37, 1.

Gardner, L. U., Dworski, M., and Delahant, A. B. (1944). J. industr. Hyg., 26, 211.

- and Cummings, D. E. (1931). Ibid., 13, 65.

Giroux, M. (1947). Sem. Hôp. Paris, 23, 1814.

Gloyne, S. R. (1938). In Lanza, A. J., Silicosis and Asbestosis, pp. 225-253. Oxford University Press.

King, E. J., Clegg, J. W., and Rae, V. M. (1946). Thorax, 1, 188.

Lanza, A. J. (1938). Silicosis and Asbestosis. Oxford University Press.

McDonald, S. (1927). Brit. med. J., 2, 1025.

Mavrogordato, A. E. Cited by Simson, F. W. (1929). Ibid., 1, 885.

Schuster, N. H. (1931). J. Path. Bact., 34, 751. 